\title{
Trial design and rationale for APOLLO, a Phase 3, placebo-controlled study of patisiran in patients with hereditary ATTR amyloidosis with polyneuropathy
}

David Adams ${ }^{1 *}$, Ole B. Suhr ${ }^{2}$, Peter J. Dyck ${ }^{3}$, William J. Litchy ${ }^{3}$, Raina G. Leahy ${ }^{4}$, Jihong Chen ${ }^{4}$, Jared Gollob ${ }^{4}$ and Teresa Coelho ${ }^{5}$

\begin{abstract}
Background: Patisiran is an investigational RNA interference (RNAi) therapeutic in development for the treatment of hereditary ATTR (hATTR) amyloidosis, a progressive disease associated with significant disability, morbidity, and mortality.

Methods: Here we describe the rationale and design of the Phase 3 APOLLO study, a randomized, double-blind, placebo-controlled, global study to evaluate the efficacy and safety of patisiran in patients with hATTR amyloidosis with polyneuropathy. Eligible patients are 18-85 years old with hATTR amyloidosis, investigator-estimated survival of $\geq 2$ years, Neuropathy Impairment Score (NIS) of 5-130, and polyneuropathy disability score $\leq$ Illb. Patients are randomized 2:1 to receive either intravenous patisiran $0.3 \mathrm{mg} / \mathrm{kg}$ or placebo once every 3 weeks. The primary objective is to determine the efficacy of patisiran at 18 months based on the difference in the change in modified NIS+7 (a composite measure of motor strength, sensation, reflexes, nerve conduction, and autonomic function) between the patisiran and placebo groups. Secondary objectives are to evaluate the effect of patisiran on Norfolk-Diabetic Neuropathy quality of life questionnaire score, nutritional status (as evaluated by modified body mass index), motor function (as measured by NIS-weakness and timed 10-m walk test), and autonomic symptoms (as measured by the Composite Autonomic Symptom Score-31 questionnaire). Exploratory objectives include assessment of cardiac function and pathologic evaluation to assess nerve fiber innervation and amyloid burden. Safety of patisiran will be assessed throughout the study.
\end{abstract}

Discussion: APOLLO represents the largest randomized, Phase 3 study to date in patients with hATTR amyloidosis, with endpoints that capture the multisystemic nature of this disease.

Trial registration: This trial is registered at clinicaltrials.gov (NCT01960348); October 9, 2013.

Keywords: Patisiran, APOLLO, RNA interference, hATTR amyloidosis, mNIS+7, Methods, Polyneuropathy

\section{Background}

Hereditary ATTR (hATTR) amyloidosis, formerly known as familial amyloid polyneuropathy (FAP), is a progressive, life-threatening disease caused by misfolded transthyretin (TTR) protein that accumulates as amyloid fibrils in multiple organs, including the nerves, heart, and gastrointestinal tract $[1,2]$. hATTR amyloidosis is a multisystemic disease with a heterogeneous clinical

\footnotetext{
* Correspondence: david.adams@aphp.fr; adams.david@neuf.fr

${ }^{1} \mathrm{CHU}$ Hôpital Bicêtre, Le Kremlin-Bicêtre CEDEX, Paris, France

Full list of author information is available at the end of the article
}

presentation, including sensory, motor, autonomic, and cardiac symptoms that are often concurrent [3-6]. The unrelenting disease course begins with unimpaired ambulation (FAP stage 1 [7]), then requirement for assistance with ambulation (FAP stage 2), which proceeds to wheelchair confinement (FAP stage 3), with patients experiencing a range of life-impacting symptoms that include burning neuropathic pain, loss of sensation in hands and feet, diarrhea/constipation, sexual impotence, and dizziness/fainting [8-10]. The median survival for 
patients with hATTR amyloidosis with polyneuropathy is reported as 5-15 years from diagnosis [9, 11-13].

To date $>120$ TTR mutations have been reported [14]; some mutations are more strongly associated with polyneuropathy (e.g. V30M [8]), and others with cardiomyopathy (e.g. V122I [15]). However, this genotype-phenotype association likely represents an over-simplification, with wide variation in presentation reported between genotypes and a mixed phenotype commonly observed $[3,16]$.

Effectively quantifying the disease burden in hATTR amyloidosis remains challenging, as there is no single test that captures the constellation of symptoms and the multisystemic nature of the condition. Indeed, even assessment of all signs and symptoms of polyneuropathy requires numerous measures, which recent data suggest are not adequately captured in current tests [17]. For example, the Neuropathy Impairment Score (NIS)-Lower Limb (NIS-LL) is based on examination of lower limbs only, so dysfunction occurring in other areas as the disease progresses cannot be captured. The widely used NIS overcomes this limitation through clinical examination of lower limbs, upper limbs, and cranial nerves, although this tool does not include nerve conduction scores, which are critical to assess the axonal neuropathy which progresses during the disease course $[6,8,12]$. In addition, the NIS does not adequately address sensory loss over the body, which is a hallmark of the disease [18]. Ultimately, scoring the full range of neuropathic impairment likely requires a combined measure of the type, severity, and distribution of neurologic signs and symptoms [17]. There is also a limited set of tools that have demonstrated utility in assessment of quality of life (QoL) and physical functioning in hATTR amyloidosis, primarily the Norfolk Quality of Life-Diabetic Neuropathy (QOL-DN), EuroQoL 5-Dimensions (EQ-5D ${ }^{\mathrm{rm}}$ ), and Short-Form 36 (SF-36) Health Survey questionnaires [19-24]. Consequently, the need remains for clinical trials that include a comprehensive set of measures to investigate disease progression and the effects of treatment on patient well-being and function.

As TTR is produced predominantly in the liver $[25,26]$ orthotopic liver transplantation (OLT) is a wellrecognized and effective treatment strategy for replacing mutant TTR protein $[27,28]$. However, OLT is only recommended for patients with early-stage hATTR amyloidosis, and survival varies according to modified body mass index (mBMI), disease duration/severity, and TTR mutation [8, 28, 29]. The procedure itself is also limited by issues such as cost, donor availability, cardiac involvement, and toxicities associated with immunosuppression $[2,30]$. Furthermore, disease progression can continue after OLT as a result of amyloid fibril deposition from wild-type TTR [31, 32]. More recently, the pharmacotherapies tafamidis and diflunisal, which stabilize the TTR complex and prevent protein misfolding (TTR tetramer stabilizers), have been utilized. Tafamidis was approved in Europe for patients with hATTR amyloidosis with earlystage neurologic disease (FAP stage 1), despite limited data, with authorization under "exceptional circumstances" due to the clinical unmet need [33]. Subsequent approvals for treatment of symptomatic polyneuropathy have followed in regions of Latin America and Asia. Diflunisal, a non-steroidal anti-inflammatory drug, has been used off label for management of hATTR amyloidosis [34]. In clinical studies, both compounds have slowed progression of neurologic impairment and were generally well tolerated [19, 24, 35]. However, progression of neuropathy symptoms or disability is still observed in some patients [24, 35-37], and tafamidis may have reduced efficacy in patients with more severe disease [38]. Thus, there remains a need for novel treatment options for hATTR amyloidosis.

Patisiran is an investigational RNA interference (RNAi) therapeutic in clinical development for hATTR amyloidosis. Patisiran is a small interfering RNA (siRNA) that targets a sequence of mRNA conserved across wild-type and all TTR variants and can thereby reduce serum levels of both wild-type and pathogenic (mutated) protein [39]. It is formulated as lipid nanoparticles which direct it to the liver, the primary source of circulating TTR [39]. The benefits of lowering mutant TTR levels in patients with hATTR amyloidosis have been demonstrated by OLT [40, 41], and data from other amyloidoses show that clinical outcomes can be improved by reducing amyloidogenic protein [42-44]. In a Phase 2 study (NCT01617967) of 29 patients with hATTR amyloidosis, two doses of patisiran $0.3 \mathrm{mg} / \mathrm{kg}$ every 3 weeks reduced mean serum TTR levels by approximately 80\% [45]. This potent TTR knockdown was observed for both wild-type and mutant (V30M) forms of TTR, and in patients who were concurrently receiving TTR tetramer stabilizers [45]. An ongoing open-label extension study (NCT01961921) has provided encouraging data, with stable measures of neuropathy impairment and patisiran being generally well tolerated through 24 months of treatment [46].

Collectively, these data support the hypothesis that TTR reduction has the potential to stabilize the progression of - or even reverse - neuropathy in patients with hATTR amyloidosis. To date, APOLLO is the largest Phase 3 clinical study in patients with hATTR amyloidosis, designed to assess the safety and efficacy of patisiran on neurologic function and QoL.

\section{Methods}

\section{Study oversight}

This study is conducted according to the guidelines of the International Conference on Harmonisation, the 
World Health Organization's Declaration of Helsinki, and the Health Insurance Portability and Accountability Act of 1996. Written informed consent is obtained from all patients who participate in the study, prior to assessment of eligibility. The study protocol was approved by the local Institutional Review Boards and Ethics Committees, and all subsequent protocol amendments underwent the same approval procedure. A clinical monitor, as a representative of Alnylam, will follow the study through periodic site visits and frequent telephone/written contact. A Data Monitoring Committee will be implemented for the study and provide independent evaluation to ensure patient safety. This trial is registered at www.clinicaltrials.gov (NCT01960348).

\section{Study overview and setting}

APOLLO is a randomized, multicenter, international, double-blind, placebo-controlled, Phase 3 study designed to evaluate the efficacy of patisiran and establish the safety of chronic dosing in adult patients with symptomatic hATTR amyloidosis with polyneuropathy. A study schematic is shown in Fig. 1. Patients are recruited from 46 sites across 19 countries (United States, France, Taiwan, Spain, Japan, Germany, Mexico, Portugal, South Korea, Sweden, Bulgaria, Italy, Canada, Turkey, Cyprus, Brazil, Netherlands, United Kingdom, and Argentina). All but two of the sites are academic hospitals.

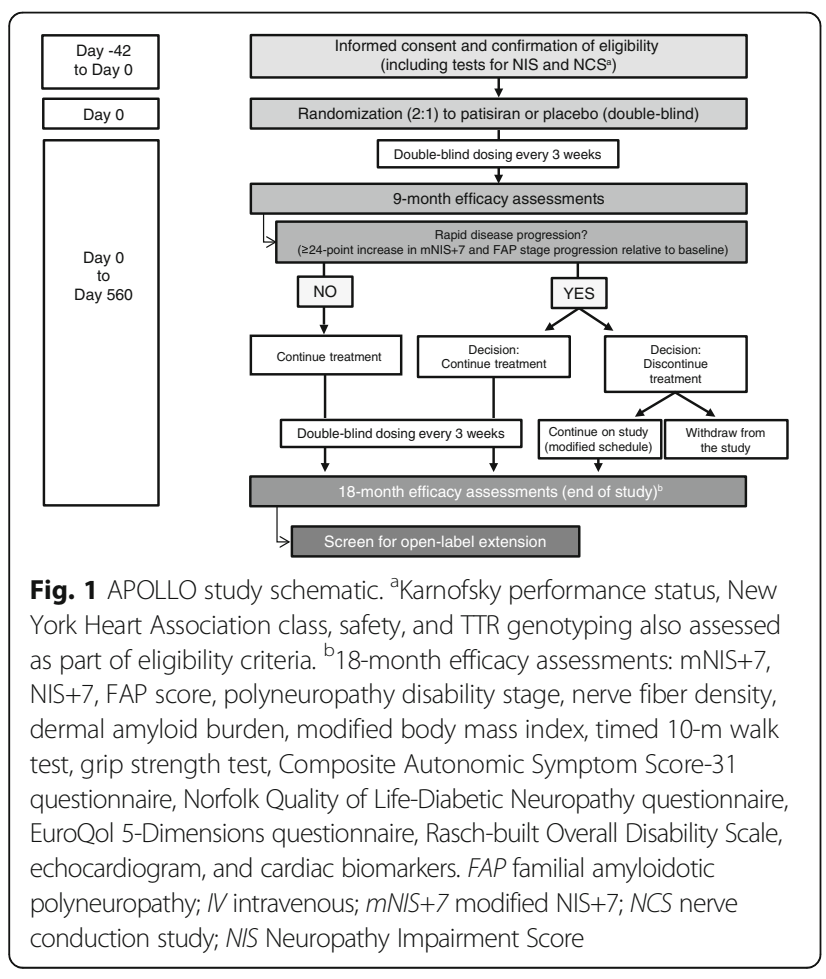

\section{Eligibility}

At screening, patients are 18-85 years old, with a diagnosis of hATTR amyloidosis, a documented TTR mutation, and investigator-estimated survival of $\geq 2$ years. Eligible patients have an NIS of 5-130, polyneuropathy disability (PND) score $\leq \mathrm{IIIb}$, and adequate biochemical liver function. The NIS range of 5-130 was selected to include patients with disease sufficiently advanced to show progression in the placebo group, but not so advanced as to preclude detection of a change in disease status. Patients with previous OLT or sensorimotor/ autonomic neuropathy due to other known causes are not included in the study. The key inclusion and exclusion criteria are summarized in Table 1.

\section{Study design}

Patients are randomized to receive either patisiran $0.3 \mathrm{mg} / \mathrm{kg}$ or placebo (normal saline $0.9 \%$ ) once every 3 weeks for 18 months. Study drug is administered as an intravenous infusion over $70 \mathrm{~min}(1 \mathrm{~mL} / \mathrm{min}$ for the first $15 \mathrm{~min}$, then $3 \mathrm{~mL} / \mathrm{min}$ thereafter).

Patients have the option of discontinuing study drug if they experience a protocol-defined rapid disease progression at 9 months, defined as $\geq 24$-point increase in $\mathrm{mNIS}+7$ from baseline (estimated 24-point increase in $\mathrm{mNIS}+7$ score in the placebo group expected by 18 months based on natural history study [47]), and FAP stage [7] progression relative to baseline, confirmed by an external adjudication committee. Such patients may receive alternative therapy (local standard of care) and will be monitored based on a modified visit schedule if they decide to stay on study. All patients who complete the final 18-month assessment are eligible to screen for an open-label extension study of long-term patisiran treatment (NCT02510261).

\section{Premedication}

To reduce the likelihood of infusion-related reactions, patients are to receive the following premedications or equivalent at least $60 \mathrm{~min}$ before each study drug infusion: dexamethasone; oral acetaminophen/paracetamol; an $\mathrm{H}_{2}$ blocker (e.g., ranitidine or famotidine); and an $\mathrm{H}_{1}$ blocker (e.g., diphenhydramine).

\section{Concomitant medications}

The use of tafamidis, diflunisal, doxycycline, tauroursodeoxycholic acid, or any investigational agent other than patisiran is prohibited during study participation. These agents may have been used before screening, but a wash-out period is mandated (14 days for tafamidis, doxycycline, or tauroursodeoxycholic acid; 3 days for diflunisal). Palliative and supportive-care medications are permitted during the study. 
Table 1 Key inclusion and exclusion criteria for the APOLLO study

\begin{tabular}{ll}
\hline Inclusion criteria & Exclusion criteria \\
\hline - Diagnosis of hATTR amyloidosis with documented mutation & - Previous liver transplantation, or liver transplantation planned during \\
- Anticipated survival $\geq 2$ years & study period \\
- Aged $18-85$ years (inclusive) & - Sensorimotor or autonomic neuropathy not related to hATTR amyloidosis \\
- NIS of 5-130 & - Primary or leptomeningeal amyloidosis \\
- PND score $\leq$ IIIb & - Type 1 diabetes \\
- NCS sum of the sural SNAP, tibial CMAP, ulnar SNAP, ulnar & - Type 2 diabetes for $\geq 5$ years \\
CMAP, and peroneal CMAP of $\geq 2$ points & - Active hepatitis B or C, or HIV infection \\
- Karnofsky performance status $\geq 60 \%$ & - NYHA heart failure classification $>2$ \\
- Absolute neutrophil count $\geq 1500$ cells $/ \mathrm{mm}^{3}$ & - Acute coronary syndrome within past 3 months \\
- Platelet count $\geq 50,000$ cells $/ \mathrm{mm}^{3}$ & - Uncontrolled cardiac arrhythmia or unstable angina \\
- Adequate biochemical liver function & - Severe reaction to a liposomal product or hypersensitivity to oligonucleotides \\
- Serum creatinine $\leq 2 \times$ ULN & - Unable to take premedications \\
& - Received an investigational agent within 30 days $/ 5$ half-lives (whichever is \\
& - Conger) of study drug administration \\
& - Currently taking tafamidis, diflunisal, doxycycline, or tauroursodeoxycholic acid
\end{tabular}

CMAP compound muscle action potential; $h A T T R$ amyloidosis hereditary transthyretin-mediated amyloidosis; NCS nerve conduction study; NIS Neuropathy Impairment Score; NYHA New York Heart Association; PND polyneuropathy disability; SNAP sensory nerve action potential; ULN upper limit of normal

${ }^{a}$ Aspartate transaminase and alanine transaminase levels $\leq 2.5 \times$ ULN; total bilirubin levels within normal limits; international normalized ratio $\leq 2.0$

\section{Stratification, randomization, and blinding}

Patients are randomized in a 2:1 (patisiran:placebo) ratio using an interactive response system. Treatment arms are balanced at study entry for: NIS (5-49 vs 50-130), earlyonset V30M disease (age $<50$ years at onset) vs all other mutations (including late-onset V30M), and previous use of tafamidis or diflunisal. Patients and study personnel who monitor patients during infusions and perform clinical assessments are blinded to the study treatment. Unblinded personnel and pharmacists prepare the drug for administration, but are not involved in patient management or safety or efficacy assessments. Details of patients who discontinue study drug at 9 months due to rapid disease progression remain blinded throughout the study.

\section{Efficacy assessments}

\section{Primary outcome measure: $\mathrm{mNIS}+7$}

The primary objective is to determine the difference between the patisiran and placebo groups in change from baseline in mNIS+7 at 18 months (Table 2). The mNIS+7 used in APOLLO has been modified from the original NIS+7 to better characterize and quantify sensation all over the body, autonomic function, and nerve conduction changes associated with hATTR amyloidosis progression [17]. A summary of the scoring components of the NIS+7 and $\mathrm{mNIS}+7$ is provided in Table 3.

The mNIS+7 assessment tool [17] used in this study is a 304-point composite measure of neurologic impairment that includes: neurologic examination of lower limbs, upper limbs, and cranial nerves (NIS-weakness and reflexes); electrophysiologic measures of small and large nerve fiber function (including nerve conduction studies [NCS] $\Sigma 5$ of ulnar, peroneal, and tibial compound muscle action potential [CMAP] amplitudes and sural and ulnar sensory nerve action potential [SNAP] amplitudes; and smart somatotopic quantitative sensory testing [S ST QSTing; including touch pressure and heat pain]) at defined body surface locations (Fig. 2); and autonomic function (postural hypotension). Scoring for NCS and postural hypotension is based on grading of function: normal $(<95$ th percentile $)=0$ points; mildly reduced $(\geq 95$ th to $<99$ th percentile $)=1$ point; and very reduced $(\geq 99$ th percentile $)=2$ points. The mNIS +7 is measured in replicates during the pre-randomization phase (at screening/baseline [ 21 days before the first dose of study drug] and at baseline [ $\geq 24 \mathrm{~h}$ after screening/baseline measure]), and at 9 and 18 months. Two independent assessments are taken at each time point, performed at least $24 \mathrm{~h}$ apart, but no greater than 7 days apart. An increase in mNIS+7 score, or in any of its components, indicates worsening impairment.

To standardize the efficacy assessment and minimize variability across the multiple centers, neuromuscular physicians were trained to perform the mNIS+7 evaluation at a central center (Dyck Peripheral Nerve Research Laboratory, Mayo Clinic, Rochester, MN, USA).

\section{Secondary and exploratory clinical outcome measures}

The secondary and exploratory objectives are to determine the effect of patisiran on a variety of clinical parameters, based on their change from baseline to 18 months (Table 2). Unless stated, parameters are assessed at screening/baseline, baseline, 9, and 18 months.

\section{Neurologic and cardiac function}

Neurologic function is measured using the NIS+7 tool [48] based on two independent readings as described for mNIS +7. For both mNIS+7 and NIS+7, the individual components of the scores will be reported. Specific motor function assessments include the timed $10-\mathrm{m}$ walk test and hand grip strength test (dynamometer), with measurements performed on separate days. Cardiac function is assessed through echocardiograms and cardiac biomarkers (troponin I and N-terminal pro-brain-type natriuretic peptide). 
Table 2 Study objectives

\begin{tabular}{|c|c|}
\hline & \\
\hline Primary & $\begin{array}{l}\text { - Determine the efficacy of patisiran by } \\
\text { evaluating the difference between the patisiran } \\
\text { and placebo groups in the change from } \\
\text { baseline of mNIS+7 at } 18 \text { months }\end{array}$ \\
\hline $\begin{array}{l}\text { Secondary } \\
\text { (hierarchical ordering) }\end{array}$ & $\begin{array}{l}\text { - Norfolk Quality of Life-Diabetic Neuropathy } \\
\text { questionnaire } \\
\text { - NIS-weakness score } \\
\text { - Level of disability (Rasch-built Overall Disability } \\
\text { Scale) } \\
\text { - Timed 10-m walk test } \\
\text { - mBMI } \\
\text { - Composite Autonomic Symptom Score } \\
\text { questionnaire }\end{array}$ \\
\hline Exploratory & $\begin{array}{l}\text { - NIS }+7 \text { score } \\
\text { - Grip strength } \\
\text { - EuroQol 5-Dimensions questionnaire } \\
\text { (EQ-5D-5L index, EQ VAS) } \\
\text { - Large versus small nerve fiber function }{ }^{b} \\
\text { - Sensory and autonomic innervation and } \\
\text { analysis of nerve fiber density and sweat gland } \\
\text { nerve fiber density } \\
\text { - Dermal amyloid content on skin biopsy } \\
\text { - FAP stage and PND score } \\
\text { - Cardiac measures (echocardiogram, troponin I, } \\
\text { and NT-proBNP levels) } \\
\text { - Pharmacodynamic biomarkers } \\
\text { (TTR, RBP, and vitamin A) } \\
\text { - Proportion of patients with rapid disease } \\
\text { progression at } 9 \text { months } \\
\text { - Lower limb nerve injury via voluntary magnetic } \\
\text { resonance neurography } \\
\text { - Columbia-Suicide Severity Rating Scale (C-SSRS) }\end{array}$ \\
\hline
\end{tabular}

FAP familial amyloidotic polyneuropathy; $m B M I$ modified body mass index; mNIS modified Neuropathy Impairment Score; NIS Neuropathy Impairment Score; NT-proBNP N-terminal pro-brain-type natriuretic peptide; PND polyneuropathy disability; $R B P$ retinol-binding protein; $T T R$ transthyretin ${ }^{a} \mathrm{mBMl}$ calculated as $\mathrm{kg} / \mathrm{m}^{2} \times$ albumin $(\mathrm{g} / \mathrm{L})$

${ }^{b}$ Nerve fiber function assessed through nerve conduction studies of 5 attributes ( $\Sigma 5)$ : quantitative sensory testing by body surface area including touch pressure and heat pain, vibration detection threshold, heart rate response to deep breathing, and postural blood pressure

\section{Quality of life/symptoms and health status}

QoL assessments include the Norfolk QOL-DN questionnaire [49], a 35-item measure sensitive to small fiber, large fiber, and autonomic nerve function, which has been shown to be a reliable indicator of disease severity in patients with hATTR amyloidosis [20] (increase in score $=$ worsening $\mathrm{QoL})$, and the EQ-5D $\mathrm{D}^{\mathrm{mo}}$ questionnaire [50] (decrease in score $=$ worsening QoL). Autonomic symptoms are evaluated using the 31-question Composite Autonomic Symptom Score (COMPASS)-31 questionnaire, which covers six autonomic domains (orthostatic intolerance, vasomotor, secretomotor, gastrointestinal, bladder, and pupillomotor) [51] (increase in score = worsening symptoms). Activity and social function are assessed through the Rasch-built Overall Disability Scale (R-ODS), a 24-item scale to capture limitations on everyday activities [52] (decrease in score $=$ worsening disability). Nutritional status is gauged using $\mathrm{mBMI}\left(\mathrm{kg} / \mathrm{m}^{2} \times\right.$ albumin $[\mathrm{g} /$ L]), taken at baseline, and days 84, 189, 357, 462, and 546 (18-month assessment). Changes in ambulation are assessed according to FAP stage [7] and PND score [53] (increase in stage/score $=$ worsening impairment), and the timed $10-\mathrm{m}$ walk test (increase in duration $=$ worsening impairment). Lower limb nerve injury is serially evaluated via voluntary magnetic resonance neurography approximately every 6 months in patients providing voluntary consent.

\section{Pathologic nerve fiber and amyloid evaluation}

Nerve fibers and amyloid deposits in the skin are quantified through measurement of intraepidermal nerve fiber density, sweat gland nerve fiber density, and dermal amyloid burden using tandem $3 \mathrm{~mm}$ skin punch biopsies in patients providing voluntary consent. At each time point, one set of biopsies is taken from the lower leg and another from the distal thigh.

\section{Pharmacodynamic assessments}

Levels of serum TTR protein, vitamin A, and retinolbinding protein (RBP) are measured at baseline, pre-dose on day 0 , and on days $21,126,252,253-272$ (9 months), $273,399,546$, and $553-560$ (18 months). Serum TTR is measured using an enzyme-linked immunosorbent assay and a turbidimetric assay. Serum RBP is quantified using nephelometry. Serum samples are evaluated by a highperformance liquid chromatography assay to determine vitamin A levels. Pharmacodynamic samples are not taken at 18 months for patients who discontinue treatment at 9 months (modified schedule).

\section{Pharmacokinetic assessments}

Plasma samples for pharmacokinetic analysis are taken predose and post-dose on days $0,21,126,252,399$, and 546 . Pharmacokinetic parameters for plasma siRNA are evaluated using a validated ATTO $^{\mathrm{mm}}$-probe high-performance liquid chromatography assay.

\section{Safety assessments}

Adverse events (AEs) are assessed throughout the study, and reported according to the Medical Dictionary of Regulatory Activities (version 18.0 or later). AEs are graded based on their severity (mild, moderate, or severe) and the causal relationship to study drug or premedication recorded. Clinical laboratory and chemistry tests, thyroid function parameters, urinalysis, anti-drug antibodies, electrocardiograms, physical and vital signs, and ophthalmology examinations (including electroretinography) are also monitored. The Columbia-Suicide Severity Rating Scale (C-SSRS) is used to assess mental status as it relates to suicidal ideation and behavior. 


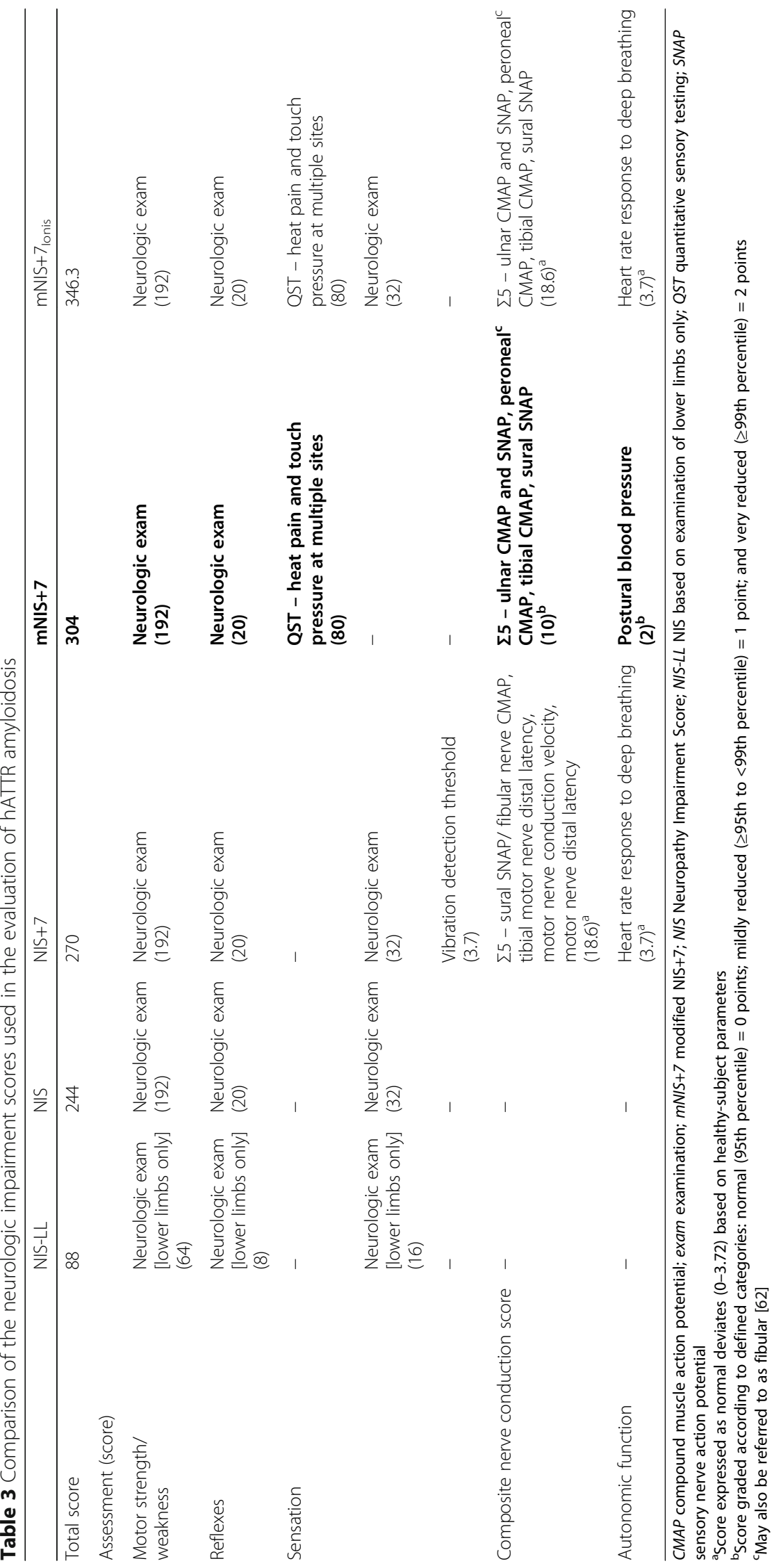




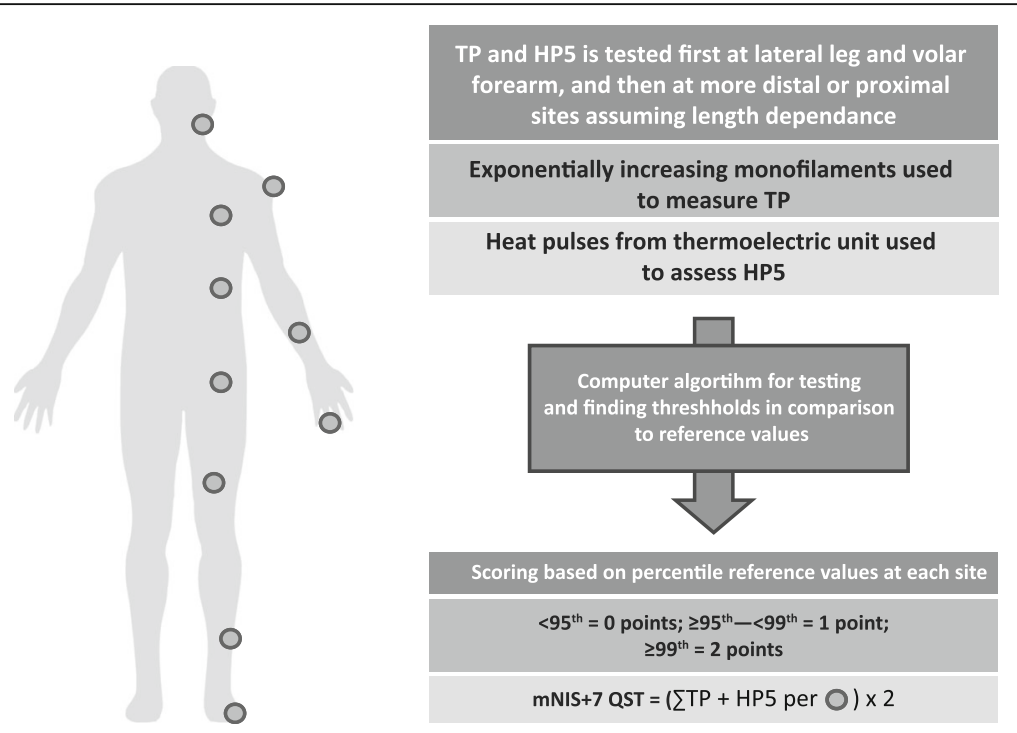

Fig. 2 Schematic representation of smart QSTing of body sites. Smart testing uses defined and quantitated stimuli of touch pressure and heat as pain using validated computer software, standard conditions, and previously obtained reference values from the assessment of healthy-subject cohorts. Dots indicate testing site. HP5, heat as pain 5 (graded 1-10); mNIS+7 modified NIS+7; NIS Neuropathy Impairment Score; S ST QSTing Smart Somatotopic Quantitative Sensory Testing; TP touch pressure [17, 62]

\section{Statistical analyses}

A sample size of approximately 200 patients will provide $90 \%$ power to test a treatment difference of 8.95 points $(37.5 \%)$ in $\mathrm{mNIS}+7$ change from baseline (primary endpoint) with a two-sided $\alpha=0.05$. The sample size of approximately 200 is based on an assumed premature discontinuation rate of $25 \%$.

The populations analyzed in the study are: the modified intent-to-treat (mITT) population (all patients who were randomized and received at least one dose of study drug); the per-protocol population (all patients who completed all efficacy assessments and did not have any major protocol violations); and the safety population (all patients who received at least one dose of study drug).

The analysis of efficacy endpoints will be conducted for the mITT population. Formal statistical hypothesis testing will be performed on the primary and secondary efficacy endpoints with all tests conducted at the nominal two-sided, 0.05 level of significance.

\section{Discussion}

The APOLLO study examines the efficacy and safety of the investigational RNAi therapeutic patisiran in a broad hATTR amyloidosis population, including patients with any amyloidogenic TTR mutation and with a wide range of neuropathy severity and age at baseline. The composite primary endpoint of APOLLO $(\mathrm{mNIS}+7)$ is well suited for use in hATTR amyloidosis because it encompasses multiple aspects of the polyneuropathy and can capture changes during disease progression. In addition,
QoL, motor function, health status, autonomic symptoms, cardiac assessments, and everyday functioning are included as secondary or exploratory endpoints to assess the impact of patisiran on a range of disease involvement.

The mNIS+7 used to assess neurologic impairment in this study is more robust and comprehensive than the tools used in the trials of tafamidis (NIS-LL) [19] or diflunisal $(\mathrm{NIS}+7)$ [24], with the modifications outlined in Table 3. One of the key attributes of the $\mathrm{mNIS}+7$ versus NIS+7 is the measurement of sensation, with S ST QSTing used in preference to NIS-sensation evaluation and vibration detection threshold. Indeed, the NISsensation score is not included in mNIS+7 in APOLLO, as the study of Suanprasert et al. in 97 patients with hATTR amyloidosis suggested that this score did not adequately capture sensation loss [17]. Compared with NIS-sensation, S ST QSTing provides an improved balance between large and small sensory nerve fibers and measures sensation loss over the whole body rather than at distal sites such as big toes and fingers. Whilst S ST QSTing is time-consuming and requires specialist training and standardized protocols to avoid procedural variability [54, 55], these demands are necessary to assess sensation loss somatopically and accurately [56]. Aside from sensation, the other major alteration from the NIS+7 is in the measurement of nerve conduction. Here, the combined NCS $\Sigma 5$ in the mNIS+7 includes only action potential amplitudes, which are more suited to capture changes in disease course for hATTR amyloidosis as this disease has a primarily axonal pathophysiology. A further change from 
the NIS +7 is the new autonomic measure: postural hypotension was included because heart rate decrease with deep breathing was considered an inconsistent assessment of autonomic function as it is unevaluable in patients with cardiac pacemakers and frequently unevaluable in patients with arrhythmias [17, 56]. It is recognized however, that pharmacologic interventions (e.g., fludrocortisone and midodrine) can be used to treat postural hypotension [57], and the use of any such strategies should be considered when gauging the effect of patisiran on this measure. With the modifications to the NIS +7 , the total score was increased to 304 points for the $\mathrm{mNIS}+7$ used in this study. Measurement of muscle weakness and stretch reflexes were considered adequate with the original $\mathrm{NIS}+7$, so these elements have not been modified [17].

However, it should be noted that the mNIS+7 described by Suanprasert et al., which is used in APOLLO, is different from that currently being used in the study investigating the anti-TTR antisense oligonucleotide IONIS-TTR $_{\mathrm{Rx}}\left(\mathrm{mNIS}+7_{\text {Ionis }}\right)$. The critical difference between these two versions of the mNIS+7 is in the measurement of sensation: $\mathrm{mNIS}+7_{\text {Ionis }}$ includes the NISsensation testing score from the original NIS+7, in addition to S ST QSTing [56] (Table 3). As discussed, NIS-sensation may not provide a true reflection of sensation impairment, with inclusion of the original NIS+7sensation testing potentially being redundant. Indeed, this may lead to double counting for assessments of sensation. The other notable difference is that the $\mathrm{mNIS}+7_{\text {Ionis }}$ does not include postural hypotension, and instead retains the heart rate decrease with deep breathing measure of the NIS+7 for assessment of autonomic function, which has the limitations discussed above for patients with pacemakers or cardiac arrhythmias. Both mNIS +7 and mNIS $+7_{\text {Ionis }}$ include only CMAPs and SNAPs as nerve conduction measures (Table 3). Regarding assessment of NCS, it is worth noting that these are expressed as normal deviates in the $\mathrm{mNIS}+7_{\text {Ionis, }}$ whereas they are graded by defined categories in mNIS +7 (Table 3).

The features of the mNIS+7 described above have been introduced to create a tool suitable for use in hATTR amyloidosis clinical studies. As assessment of neuropathic measures is subject to variability between investigators, extensive training is provided to support the use of a standardized and validated methodology and ensure that scoring is consistent and accurate. Specifically, specialized training is provided for neuromuscular physicians, with certification upon completion. For clinical assessment of neuropathy signs and symptoms, neuromuscular experts are trained to use only unequivocal abnormalities (accounting for age, sex, physical fitness, and anthropomorphic variables) rather than more traditional clinical criteria, and not to grade for concomitant neuromuscular disease. Previous analyses have shown that this strategy, when used by trained specialists, leads to increased reproducibility and a notable improvement in proficiency when scoring clinical measures, such as weakness and reflexes, as used in APOLLO [58, 59]. Variability between centers has also been noted for NCS, which is countered in this study through specialist training on techniques and reference values, and evaluation of tracings at a central reading center; these methodologies have been shown to reduce inter-investigator variability [60, 61]. Of the other mNIS+7 measures, the S ST QSTings used in APOLLO are also standardized and referenced, to ensure generation of accurate and comparable data across the study [62].

The clinical relevance of NIS has been previously demonstrated, with total NIS correlated with FAP stage, PND score, and QoL (Norfolk QOL-DN and SF-36) in patients with hATTR amyloidosis $[47,56]$. In addition, rapid worsening of NIS was observed in untreated patients in a natural history study of patients with hATTR amyloidosis [47], fitting with the relentless progression expected for this disease [8]. The estimated rate of NIS progression for a patient with a baseline NIS of 32 was 14.3 points per year [47], and a separate analysis has indicated a worsening in NIS-LL of up to 7.4 points over 12 months in patients with a baseline NIS-LL of 20-30 [63]. The rapid neurologic deterioration observed in patients with hATTR amyloidosis contrasts with lower rates of NIS progression seen in other neuropathies $[64,65]$. For example, in patients with mild-to-moderate diabetic distal symmetric sensorimotor polyneuropathy, NIS and NIS-LL increased by only 0.61 and 0.43 points, respectively, over 4 years of placebo treatment [64]. Furthermore, a study of disease progression in CharcotMarie-Tooth disease type $1 \mathrm{~A}$ reported an annual NIS increase of 1.37 points [65].

Based on the described modifications to the NIS, it is anticipated that the mNIS+7 will more accurately capture the degree of polyneuropathy and neurologic impairment in patients with hATTR amyloidosis. Indeed, rapid disease progression in untreated patients has also been demonstrated using the $\mathrm{mNIS}+7$, with an increase of approximately 24 points anticipated after 18 months [47]. The clinical value of the mNIS +7 was further demonstrated in recent analyses of the baseline data from APOLLO, which showed an association between $\mathrm{mNIS}+7$ and FAP stage and PND score (Fig. 3). In addition, investigation of the $\mathrm{mNIS}+7_{\text {Ionis }}$ showed that clinical polyneuropathy signs and symptoms correlated strongly with assessments of action potential amplitudes and somatotopic touch pressure [56], which are also included in the mNIS +7 . Of the other measures taken in 

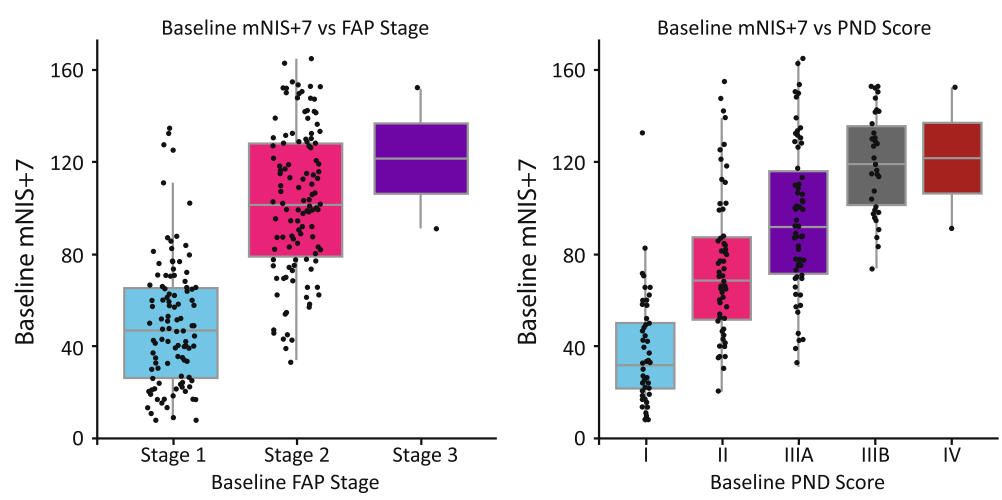

Fig. 3 Association between mNIS+7 and (A) FAP stage and (B) PND score using baseline data from the APOLLO study $(n=225)$. FAP familial amyloidotic polyneuropathy; $m N I S+7$ modified Neuropathy Impairment Score +7 ; PND polyneuropathy disability

APOLLO, a correlation between mNIS+7 and TTR knockdown was demonstrated in the Phase 2 openlabel extension study of patisiran [66], supporting the hypothesis that reducing levels of amyloidogenic protein leads to clinical benefit in hATTR amyloidosis (e.g., via OLT [28]).

hATTR amyloidosis is associated with symptoms across multiple systems $[2,67]$. In addition to the neurologic symptoms, patients often present with a mixed phenotype including concurrent cardiac symptoms. Cardiomyopathy associated with hATTR amyloidosis can lead to heart failure and death, highlighting the need for vigilance around cardiac amyloid fibril accumulation. The APOLLO study therefore includes echocardiographic and biochemical cardiac parameters to assess the impact of patisiran on cardiac progression.

The impact of the diverse disease symptoms may not be fully captured by clinical and laboratory examinations, so QoL measures were included as key endpoints in the studies of tafamidis and diflunisal [19, 24]. APOLLO uses the Norfolk QOL-DN and EQ-5D questionnaires to assess QoL. The Norfolk QOL-DN questionnaire evaluates small and large nerve fiber function in addition to autonomic impairment and activities of daily living. It has demonstrated utility in patients with hATTR amyloidosis with polyneuropathy both as a measure of disease severity and as a clinical endpoint to assess response to treatment [20,21]. The EQ-5D questionnaire has been used as part of the international Transthyretin Amyloidosis Outcomes Survey (THAOS), which demonstrated worsening QoL with disease progression [22]. Autonomic symptoms, particularly gastrointestinal events, are common in patients with hATTR amyloidosis [23] underpinning the comprehensive assessment of autonomic function using the COMPASS31 questionnaire [51]. Other measures in APOLLO include the R-ODS survey [52] for assessment of the effects of patisiran on activities of daily living. The R-ODS has already been used to demonstrate that hATTR amyloidosis can affect activities such as washing dishes or fastening buttons [68], and has been validated in patients with V30M hATTR amyloidosis [69].

The pharmacotherapies currently approved or available for hATTR amyloidosis can slow or sometimes stabilize disease, but there is little evidence that complete stabilization or reversal of nerve damage can be achieved. Sensory and autonomic innervation in skin punch biopsies are being evaluated in APOLLO to determine whether patisiran can increase nerve fiber density. Increases in sweat gland fiber density were observed in the Phase 2 open-label extension study of patisiran [46], and APOLLO will provide an opportunity for these findings to be validated in a placebo-controlled setting. Of interest, sweat gland nerve fiber density has previously been associated with ambulation in patients with hATTR amyloidosis [70]. In addition to nerve fiber density, these skin biopsies are also being evaluated in both the Phase 2 open-label extension study and APOLLO for changes in dermal amyloid burden.

Aside from clinical endpoints related to efficacy, safety and pharmacodynamics will also be assessed throughout the APOLLO study and subsequent open-label extension. Longer-term data from the Phase 2 open-label extension study suggest patisiran is well tolerated, but APOLLO will allow comparison against a placebocontrolled arm. In particular, the safety of prolonged lowering of vitamin A levels, associated with TTR reduction, will be assessed. TTR reduction itself provides a convenient biomarker to assess patisiran activity, and reduction in the pathogenic protein may also relate to clinical parameters.

Recruitment for APOLLO started in December 2013 and was completed in January 2016, with 225 patients enrolled. This trial represents the largest Phase 3 study of an RNAi strategy for the treatment of hATTR amyloidosis, with clinical endpoints that strive to assess the multiple 
ways in which this disease affects patient well-being and function. The Patisiran Global open-label extension study was initiated in July 2015, and will provide further data on long-term safety and efficacy of patisiran in patients with hATTR amyloidosis.

\begin{abstract}
Abbreviations
AE: Adverse event; CMAP: Compound muscle action potential; COMPASS-31: 31-question Composite Autonomic Symptom Score; EQ-5D: EuroQol 5Dimensions; FAP: Familial amyloid polyneuropathy; hATTR amyloidosis: Hereditary ATTR amyloidosis; mBMl: Modified body mass index; mITT: Modified intent-to-treat; mNIS+7: Modified Neuropathy Impairment Score $\mathrm{mNIS}+7_{\text {Ionis: }} \mathrm{mNIS}+7$ being used in the study investigating the anti-TTR antisense oligonucleotide IONIS-TTRRxi NCS: Nerve conduction study; NIS: Neuropathy Impairment Score; NIS-LL: NIS-Lower Limb; OLT: Orthotopic liver transplantation; PND: Polyneuropathy disability score; QoL: Quality of life; QOL-DN: Norfolk Quality of Life-Diabetic Neuropathy; RBP: Retinol-binding protein; RNAi: RNA interference; R-ODS: Rasch-built Overall Disability Scale; SF36: Short-Form 36; siRNA: Small interfering RNA; SNAP: Sensory nerve action potential; S ST QSTing: Smart somatotopic quantitative sensory testing; TTR: Transthyretin
\end{abstract}

\section{Acknowledgements}

Editorial support was provided by Adelphi Communications Ltd., UK, funded by Alnylam.

\section{Funding}

This study is funded by Alnylam Pharmaceuticals.

\section{Availability of data and materials}

Not applicable.

\section{Authors' contributions}

Authorship was decided based on ICJME criteria. DA, OS, PD, WL, RL, JC, JG, and TC were involved in the conception and design of the study. DA, OS, $P D, W L, R L, J C, J G$, and TC critically reviewed the manuscript and approved the final version. RL, JC, and JG are employed by the study funder. All authors have read and approved the final manuscript, and agreed to its publication.

\section{Correspondence information for trial sponsor}

Alnylam Pharmaceuticals Cambridge, 300 Third Street, 3rd Floor, Cambridge, MA 02142, USA. Tel: 001617551 8200; Fax: 0016175518101.

\section{Ethics approval and consent to participate}

This study is conducted according to the guidelines of the International Conference on Harmonisation, the World Health Organization's Declaration of Helsinki, and the Health Insurance Portability and Accountability Act of 1996. Written informed consent is obtained from all patients who participate in the study, prior to assessment of eligibility. The study protocol was approved by the local Institutional Review Boards and Ethics Committees, and all subsequent protocol amendments underwent the same approval procedure.

\section{Consent for publication}

Not applicable.

\section{Competing interests}

Adams: Research funding and consultancy fees from Alnylam Pharmaceuticals, and personal symposium fees from Pfizer Inc. Suhr: Personal fees and non-financial support from Alnylam Pharmaceuticals, and personal fees from Prothena Pharmaceuticals and Pfizer Inc. Dyck: Compensation for training and quality assurance associated with clinical trials from Alnylam Pharmaceuticals and IONIS Pharmaceuticals; honoraria from Diabetes journal as Associate Editor. Litchy: Compensation for travel and training of investigators from Alnylam Pharmaceuticals and IONIS Pharmaceuticals. Leahy: Employment by Alnylam Pharmaceuticals. Chen: Employment by Alnylam Pharmaceuticals. Gollob: Employment by Alnylam Pharmaceuticals. Coelho: Compensation for training from Pfizer Inc. and Alnylam Pharmaceuticals, and compensation for travel from Pfizer Inc., Alnylam Pharmaceuticals, and IONIS Pharmaceuticals.

\section{Publisher's Note}

Springer Nature remains neutral with regard to jurisdictional claims in published maps and institutional affiliations.

\section{Author details}

${ }^{1}$ CHU Hôpital Bicêtre, Le Kremlin-Bicêtre CEDEX, Paris, France. ${ }^{2}$ Department of Public Health and Clinical Medicine, Umeå University Hospital, Umeå, Sweden. ${ }^{3}$ Department of Neurology, Mayo Clinic, Rochester, MN, USA. ${ }^{4}$ Alnylam Pharmaceuticals, Cambridge, MA, USA. ${ }^{5}$ Hospital Santo António, Centro Hospitalar do Porto, Porto, Portugal.

Received: 4 April 2017 Accepted: 22 August 2017

Published online: 11 September 2017

\section{References}

1. Hanna M. Novel drugs targeting transthyretin amyloidosis. Curr Heart Fail Rep. 2014;11(1):50-7.

2. Hawkins PN, Ando Y, Dispenzeri A, Gonzalez-Duarte A, Adams D, Suhr OB Evolving landscape in the management of transthyretin amyloidosis. Ann Med. 2015;47(8):625-38.

3. Coelho T, Maurer MS, Suhr OB. THAOS - The Transthyretin Amyloidosis Outcomes Survey: initial report on clinical manifestations in patients with hereditary and wild-type transthyretin amyloidosis. Curr Med Res Opin. 2013;29(1):63-76.

4. Mohty D, Damy T, Cosnay P, Echahidi N, Casset-Senon D, Virot P, Jaccard A. Cardiac amyloidosis: updates in diagnosis and management. Arch Cardiovasc Dis. 2013;106(10):528-40.

5. Conceicao I, Gonzalez-Duarte A, Obici L, Schmidt HH, Simoneau D, Ong ML, Amass L. "Red-flag" symptom clusters in transthyretin familial amyloid polyneuropathy. J Peripher Nerv Syst. 2016;21(1):5-9.

6. Shin SC, Robinson-Papp J. Amyloid neuropathies. Mt Sinai J Med. 2012;79(6):733-48

7. Coutinho P, DeSilva A, Lima J, Barbosa A. Forty years of experience with type I amyloid neuropathy: review of 483 cases. In: Glenner G, Costa P, de Freitas A, editors. Amyloid and Amyloidosis. Amsterdam: Excerpta Medica; 1980. pp. 88-98.

8. Ando Y, Coelho T, Berk JL, Cruz MW, Ericzon BG, Ikeda S, Lewis WD, Obici L, Plante-Bordeneuve V, Rapezzi C, et al. Guideline of transthyretin-related hereditary amyloidosis for clinicians. Orphanet J Rare Dis. 2013;8:31.

9. Koike H, Tanaka F, Hashimoto R, Tomita M, Kawagashira Y, lijima M, Fujitake J, Kawanami T, Kato T, Yamamoto M, et al. Natural history of transthyretin Val30Met familial amyloid polyneuropathy: analysis of late-onset cases from non-endemic areas. J Neurol Neurosurg Psychiatry. 2012;83(2):152-8.

10. Planté-Bordeneuve V, Said G. Familial amyloid polyneuropathy. Lancet Neurol. 2011;10(12):1086-97.

11. Benson MD, Teague SD, Kovacs R, Feigenbaum H, Jung J, Kincaid JC. Rate of progression of transthyretin amyloidosis. Am J Cardiol. 2011;108(2):285-9.

12. Mariani LL, Lozeron P, Theaudin M, Mincheva Z, Signate A, Ducot B, Algalarrondo V, Denier C, Adam C, Nicolas G, et al. Genotype-phenotype correlation and course of transthyretin familial amyloid polyneuropathies in France. Ann Neurol. 2015;78(6):901-16.

13. Sattianayagam PT, Hahn AF, Whelan CJ, Gibbs SD, Pinney JH, Stangou AJ, Rowczenio D, Pflugfelder PW, Fox Z, Lachmann HJ, et al. Cardiac phenotype and clinical outcome of familial amyloid polyneuropathy associated with transthyretin alanine 60 variant. Eur Heart J. 2012;33(9):1120-7.

14. Rowczenio DM, Noor I, Gillmore JD, Lachmann HJ, Whelan C, Hawkins PN, Obici L, Westermark P, Grateau G, Wechalekar AD. Online registry for mutations in hereditary amyloidosis including nomenclature recommendations. Hum Mutat. 2014;35(9):E2403-12.

15. Ruberg FL, Berk JL. Transthyretin (TTR) cardiac amyloidosis. Circulation. 2012;126(10):1286-300.

16. Maurer MS, Hanna M, Grogan M, Dispenzieri A, Witteles R, Drachman B, Judge DP, Lenihan DJ, Gottlieb SS, Shah SJ, et al. Genotype and phenotype of transthyretin cardiac amyloidosis: THAOS (Transthyretin Amyloid Outcome Survey). J Am Coll Cardiol. 2016;68(2):161-72.

17. Suanprasert N, Berk JL, Benson MD, Dyck PJ, Klein CJ, Gollob JA, Bettencourt BR, Karsten V, Dyck PJ. Retrospective study of a TTR FAP cohort to modify NIS+7 for therapeutic trials. J Neurol Sci. 2014;344(1-2):121-8.

18. Andrade C. A peculiar form of peripheral neuropathy; familiar atypical generalized amyloidosis with special involvement of the peripheral nerves. Brain. 1952;75(3):408-27. 
19. Coelho T, Maia LF, Martins da Silva A, Waddington Cruz M, PlanteBordeneuve V, Lozeron P, Suhr OB, Campistol JM, Conceicao IM, Schmidt $\mathrm{HH}$, et al. Tafamidis for transthyretin familial amyloid polyneuropathy: a randomized, controlled trial. Neurology. 2012;79(8):785-92.

20. Vinik EJ, Vinik Al, Paulson JF, Merkies IS, Packman J, Grogan DR, Coelho T. Norfolk QOL-DN: validation of a patient reported outcome measure in transthyretin familial amyloid polyneuropathy. J Peripher Nerv Syst. 2014;19(2):104-14.

21. Coelho T, Vinik A, Vinik EJ, Tripp T, Packman J, Grogan DR. Clinical measures in transthyretin familial amyloid polyneuropathy. Muscle Nerve. 2017;55(3):323-32.

22. Inês M, Coelho T, Conceição I, Ferreira L, de Carvalho M, Costa J. Transthyretin familial amyloid polyneuropathy impact on health-related quality of life. Orphanet J Rare Dis. 2015;10(Supp. 1):O28.

23. Wixner J, Mundayat R, Karayal ON, Anan I, Karling P, Suhr OB, THAOS investigators. THAOS: gastrointestinal manifestations of transthyretin amyloidosis - common complications of a rare disease. Orphanet J Rare Dis. 2014;9:61.

24. Berk JL, Suhr OB, Obici L, Sekijima Y, Zeldenrust SR, Yamashita T, Heneghan MA, Gorevic PD, Litchy WJ, Wiesman JF, et al. Repurposing diflunisal for familial amyloid polyneuropathy: a randomized clinical trial. JAMA. 2013;310(24):2658-67.

25. Mita S, Maeda S, Shimada K, Araki S. Analyses of prealbumin mRNAs in individuals with familial amyloidotic polyneuropathy. J Biochem. 1986;100(5):1215-22

26. Holmgren G, Steen L, Ekstedt J, Groth CG, Ericzon BG, Eriksson S, Andersen O, Karlberg I, Norden G, Nakazato M, et al. Biochemical effect of liver transplantation in two Swedish patients with familial amyloidotic polyneuropathy (FAP-met30). Clin Genet. 1991;40(3):242-6.

27. Adams D, Samuel D, Goulon-Goeau C, Nakazato M, Costa PM, Feray C, Plante V, Ducot B, Ichai P, Lacroix C, et al. The course and prognostic factors of familial amyloid polyneuropathy after liver transplantation. Brain. 2000;123(Pt 7):1495-504.

28. Ericzon BG, Wilczek HE, Larsson M, Wijayatunga P, Stangou A, Pena JR, Furtado E, Barroso E, Daniel J, Samuel D, et al. Liver transplantation for hereditary transthyretin amyloidosis: after 20 years still the best therapeutic alternative? Transplantation. 2015;99(9):1847-54.

29. Suhr OB, Larsson M, Ericzon BG, Wilczek HE, on behalf of the FAPWTR's investigators. Survival after transplantation in patients with mutations other than Val30Met: extracts from the FAP World Transplant Registry. Transplantation. 2016;100(2):373-81

30. Carvalho A, Rocha A, Lobato L. Liver transplantation in transthyretin amyloidosis: issues and challenges. Liver Transpl. 2015;21(3):282-92.

31. Adams D, Buades J, Suhr O, Obici L, Coelho T. Preliminary assessment of neuropathy progression in patients with hereditary ATTR amyloidosis after orthotopic liver transplantation. Orphanet J Rare Dis. 2015; 10(Suppl. 1):P19.

32. Liepnieks JJ, Zhang LQ, Benson MD. Progression of transthyretin amyloid neuropathy after liver transplantation. Neurology. 2010;75(4):324-7.

33. EMA. Vyndaqel [http://www.ema.europa.eu/ema/index.jsp?curl=pages/ medicines/human/medicines/002294/human_med_001498.jsp\&mid= WC0b01ac058001d124].

34. Amyloidosis Patient Information Site. National Amyloidosis Centre. ATTR Anyloidosis. [http://www.amyloidosis.org.uk/introduction-to-attr-amyloidosis/ 2015].

35. Coelho T, Maia LF, da Silva AM, Cruz MW, Plante-Bordeneuve V, Suhr OB, Conceicao I, Schmidt HH, Trigo P, Kelly JW, et al. Long-term effects of tafamidis for the treatment of transthyretin familial amyloid polyneuropathy. J Neurol. 2013:260(11):2802-14

36. Plante-Bordeneuve V, Gorram F, Salhi H, Nordine T, Ayache SS, Le Corvoisier $P$, Azoulay D, Feray C, Damy T, Lefaucheur JP. Long-term treatment of transthyretin familial amyloid polyneuropathy with tafamidis: a clinical and neurophysiological study. J Neurol. 2017;264(2):268-76.

37. Cortese A, Vita G, Luigetti M, Russo M, Bisogni G, Sabatelli M, Manganelli F, Santoro L, Cavallaro T, Fabrizi GM, et al. Monitoring effectiveness and safety of Tafamidis in transthyretin amyloidosis in Italy: a longitudinal multicenter study in a non-endemic area. J Neurol. 2016;263(5):916-24.

38. Lozeron P, Theaudin M, Mincheva Z, Ducot B, Lacroix C, Adams D, French Network for FAP (CORNAMYL). Effect on disability and safety of Tafamidis in late onset of Met30 transthyretin familial amyloid polyneuropathy. Eur J Neurol. 2013;20(12):1539-45.
39. Coelho T, Adams D, Silva A, Lozeron P, Hawkins PN, Mant T, Perez J, Chiesa J, Warrington S, Tranter E, et al. Safety and efficacy of RNAi therapy for transthyretin amyloidosis. N Engl J Med. 2013;369(9):819-29.

40. Okamoto S, Wixner J, Obayashi K, Ando Y, Ericzon BG, Friman S, Uchino M, Suhr OB. Liver transplantation for familial amyloidotic polyneuropathy: impact on Swedish patients' survival. Liver Transpl. 2009;15(10):1229-35.

41. Tashima K, Ando Y, Terazaki H, Yoshimatsu S, Suhr OB, Obayashi K, Yamashita T, Ando E, Uchino M, Ando M. Outcome of liver transplantation for transthyretin amyloidosis: follow-up of Japanese familial amyloidotic polyneuropathy patients. J Neurol Sci. 1999;171(1):19-23.

42. Gillmore JD, Lovat LB, Persey MR, Pepys MB, Hawkins PN. Amyloid load and clinical outcome in AA amyloidosis in relation to circulating concentration of serum amyloid a protein. Lancet. 2001;358(9275):24-9.

43. Lachmann HJ, Gallimore R, Gillmore JD, Carr-Smith HD, Bradwell AR, Pepys MB, Hawkins PN. Outcome in systemic AL amyloidosis in relation to changes in concentration of circulating free immunoglobulin light chains following chemotherapy. Br J Haematol. 2003;122(1):78-84.

44. Gillmore JD, Stangou AJ, Tennent GA, Booth DR, O'Grady J, Rela M, Heaton ND, Wall CA, Keogh JA, Hawkins PN. Clinical and biochemical outcome of hepatorenal transplantation for hereditary systemic amyloidosis associated with apolipoprotein Al Gly26Arg. Transplantation. 2001;71(7):986-92.

45. Suhr OB, Coelho T, Buades J, Pouget J, Conceicao I, Berk J, Schmidt H, Waddington-Cruz M, Campistol JM, Bettencourt BR, et al. Efficacy and safety of patisiran for familial amyloidotic polyneuropathy: a phase II multi-dose study. Orphanet J Rare Dis. 2015;10:109.

46. Suhr O, Adams D, Coelho T, Waddington Cruz M, Schmidt H, Buades J, Campistol J, Pouget J, Berk J, Polydefkis M, et al. Phase 2 open-label extension study of patisiran, an investigational RNAi therapeutic for the tereatment of hereditary ATTR amyloidosis with polyneuropathy. In: The XVth International Symposium on Amyloidosis (International Society of Amyloidosis) Uppsala: Abstract PA80; 2016.

47. Adams D, Coelho T, Obici L, Merlini G, Mincheva Z, Suanprasert N, Bettencourt BR, Gollob JA, Gandhi PJ, Litchy WJ, et al. Rapid progression of familial amyloidotic polyneuropathy: a multinational natural history study. Neurology. 2015;85(8):675-82.

48. Dyck PJ, Herrmann DN, Staff NP, Dyck PJ. Assessing decreased sensation and increased sensory phenomena in diabetic polyneuropathies. Diabetes. 2013;62(11):3677-86.

49. Vinik EJ, Hayes RP, Oglesby A, Bastyr E, Barlow P, Ford-Molvik SL, Vinik Al. The development and validation of the Norfolk QOL-DN, a new measure of patients' perception of the effects of diabetes and diabetic neuropathy. Diabetes Technol Ther. 2005;7(3):497-508.

50. EuroQol Group. EuroQol-a new facility for the measurement of healthrelated quality of life. Health Policy. 1990;16:199-208.

51. Sletten DM, Suarez GA, Low PA, Mandrekar J, Singer W. COMPASS 31: a refined and abbreviated composite autonomic symptom score. Mayo Clin Proc. 2012;87(12):1196-201.

52. van Nes SI, Vanhoutte EK, van Doorn PA, Hermans M, Bakkers M, Kuitwaard K, Faber CG, Merkies IS. Rasch-built overall disability scale (R-ODS) for immune-mediated peripheral neuropathies. Neurology. 2011;76(4):337-45.

53. Suhr O, Danielsson A, Holmgren G, Steen L. Malnutrition and gastrointestinal dysfunction as prognostic factors for survival in familial amyloidotic polyneuropathy. J Intern Med. 1994;235(5):479-85.

54. Backonja MM, Attal N, Baron R, Bouhassira D, Drangholt M, Dyck PJ, Edwards RR, Freeman R, Gracely R, Haanpaa $M H$, et al. Value of quantitative sensory testing in neurological and pain disorders: NeuPSIG consensus. Pain. 2013;154(9):1807-19.

55. Cruz-Almeida Y, Fillingim RB. Can quantitative sensory testing move us closer to mechanism-based pain management? Pain Med. 2014;15(1):61-72.

56. Dyck PJ, Kincaid JC, Dyck PJB, Chaudhry V, Goyal NA, Alves C, Salhi H, Wiesman JF, Labeyrie C, Robinson-Papp J, et al. Assessing $\mathrm{mNIS}+7_{\text {Ionis }}$ and international neurologists' proficiency in a familial amyloidotic polyneuropathy trial. Muscle Nerve. 2017. doi:10.1002/mus.25563. [Epub ahead of print].

57. Lanier JB, Mote MB, Clay EC. Evaluation and management of orthostatic hypotension. Am Fam Physician. 2011;84(5):527-36.

58. Dyck PJ, Overland CJ, Low PA, Litchy WJ, Davies JL, Dyck PJ, O'Brien PC, Cl vs. NPhys Trial Investigators, Albers JW, Andersen $\mathrm{H}$, et al. Signs and symptoms versus nerve conduction studies to diagnose diabetic sensorimotor polyneuropathy: Cl vs. NPhys trial. Muscle Nerve. 2010;42(2):157-64 
59. Dyck PJ, Overland CJ, Low PA, Litchy WJ, Davies JL, Dyck PJ, Carter RE, Melton LJ, Andersen $\mathrm{H}$, Albers JW, et al. "Unequivocally abnormal" vs "usual" signs and symptoms for proficient diagnosis of diabetic polyneuropathy: $\mathrm{Cl}$ vs N Phys trial. Arch Neurol. 2012;69(12):1609-14.

60. Dyck PJ, Albers JW, Wolfe J, Bolton CF, Walsh N, Klein CJ, Zafft AJ, Russell $J W$, Thomas K, Davies JL, et al. A trial of proficiency of nerve conduction: greater standardization still needed. Muscle Nerve. 2013;48(3):369-74.

61. Litchy WJ, Albers JW, Wolfe J, Bolton CF, Walsh N, Klein CJ, Zafft AJ, Russell JW, Zwirlein M, Overland CJ, et al. Proficiency of nerve conduction using standard methods and reference values (cl. NPhys trial 4). Muscle Nerve. 2014;50(6):900-8.

62. Dyck PJ, Argyros B, Russell JW, Gahnstrom LE, Nalepa S, Albers JW, Lodermeier KA, Zafft AJ, Dyck PJ, Klein CJ, et al. Multicenter trial of the proficiency of smart quantitative sensation tests. Muscle Nerve. 2014:49(5):645-53.

63. Li H, Schwartz J, Keohane D. Impact of baseline neurologic score on disease progression in transthyretin familial amyloid polyneuropathy. In: International Society of Amyloidosis (ISA). Uppsala: Poster PB2; 2016.

64. Ziegler D, Low PA, Litchy WJ, Boulton AJ, Vinik Al, Freeman R, Samigullin R, Tritschler H, Munzel U, Maus J, et al. Efficacy and safety of antioxidant treatment with alpha-lipoic acid over 4 years in diabetic polyneuropathy: the NATHAN 1 trial. Diabetes Care. 2011:34(9):2054-60.

65. Shy ME, Chen L, Swan ER, Taube R, Krajewski KM, Herrmann D, Lewis RA, McDermott MP. Neuropathy progression in Charcot-Marie-tooth disease type 1A. Neurology. 2008;70(5):378-83.

66. Coelho T, Suhr O, Conceicao I, Waddington Cruz M, Schmidt H, Buades J, Campistol J, Pouget J, Berk J, Ziyadeh N, et al. Relationship between TTR knockdown and change in $\mathrm{mNIS}+7$ : preliminary correlation findings from the Phase 2 open-label extension study of patisiran, an investigational RNAi therapeutic for hereditary ATTR amyloidosis with polyneuropathy. In: The $\mathrm{XV}$ th International Symposium on Amyloidosis (Internation Society of Amyloidosis). Uppsala: Poster PA83; 2016.

67. Ueda M, Ando Y. Recent advances in transthyretin amyloidosis therapy. Transl Neurodegener. 2014;3:19.

68. Amyloidosis Foundation: Understanding the patient voice in hereditary transthyretin-mediated amyloidosis. Chicago: Amyloidosis Foundation \& Amyloidosis Support Groups Annual Meeting; 2015.

69. Pruppers MH, Merkies IS, Faber CG, Da Silva AM, Costa V, Coelho T. The Val30Met familial amyloid polyneuropathy specific Rasch-built overall disability scale (FAP-RODS(c)). J Peripher Nerv Syst. 2015;20(3):319-27.

70. Chao CC, Huang CM, Chiang HH, Luo KR, Kan HW, Yang NC, Chiang H, Lin WM, Lai SM, Lee MJ, et al. Sudomotor innervation in transthyretin amyloid neuropathy: pathology and functional correlates. Ann Neurol. 2015;78(2):272-83.

\section{Submit your next manuscript to BioMed Central and we will help you at every step:}

- We accept pre-submission inquiries

- Our selector tool helps you to find the most relevant journal

- We provide round the clock customer support

- Convenient online submission

- Thorough peer review

- Inclusion in PubMed and all major indexing services

- Maximum visibility for your research

Submit your manuscript at www.biomedcentral.com/submit

CBiomed Central 